2016-09

\title{
Logic brightens my day: Evidence for implicit sensitivity to logical validity.
}

\section{Trippas, D}

http://hdl.handle.net/10026.1/9934

10.1037/xIm0000248

Journal of Experimental Psychology: Learning, Memory, and Cognition

American Psychological Association (APA)

All content in PEARL is protected by copyright law. Author manuscripts are made available in accordance with publisher policies. Please cite only the published version using the details provided on the item record or document. In the absence of an open licence (e.g. Creative Commons), permissions for further reuse of content should be sought from the publisher or author. 


\title{
Logic Brightens My Day: Evidence for Implicit Sensitivity to Logical Validity
}

\author{
Dries Trippas \\ Max Planck Institute for Human Development, Berlin, Germany
}

\author{
Simon J. Handley and Michael F. Verde \\ Plymouth University
}

\author{
Kinga Morsanyi \\ Queen's University Belfast
}

\begin{abstract}
A key assumption of dual process theory is that reasoning is an explicit, effortful, deliberative process. The present study offers evidence for an implicit, possibly intuitive component of reasoning. Participants were shown sentences embedded in logically valid or invalid arguments. Participants were not asked to reason but instead rated the sentences for liking (Experiment 1) and physical brightness (Experiments 2-3). Sentences that followed logically from preceding sentences were judged to be more likable and brighter. Two other factors thought to be linked to implicit processing_-sentence believability and facial expression- had similar effects on liking and brightness ratings. The authors conclude that sensitivity to logical structure was implicit, occurring potentially automatically and outside of awareness. They discuss the results within a fluency misattribution framework and make reference to the literature on discourse comprehension.
\end{abstract}

Keywords: logic, beliefs, brightness, intuition, dual process theory

Supplemental materials: http://dx.doi.org/10.1037/xlm0000248.supp

The idea that logic and intuition represent different modes of thinking has deep roots in philosophy and psychology, and it continues to shape theories in many cognitive and social domains (for a review, see Evans, 2008). According to the dual process theory of reasoning, maximizing the probability of computing a normatively correct response typically requires a reasoning process that is deliberate, effortful and time-consuming. The alternative is to use heuristics that are intuitive, simple, and fast but often result in suboptimal performance (Evans, 2008; Kahneman, 2011; although see also Gigerenzer, Todd, \& the ABC Research Group, 1999, for a different perspective). For example, in situations that require deduction, people are prone to accept conclusions based on readily accessible beliefs, regardless of their logical validity (Evans, Barston, \& Pollard, 1983). Dual process theory classifies formal reasoning and the use of heuristics as Type II and Type I

This article was published Online First February 18, 2016.

Dries Trippas, Center for Adaptive Rationality, Max Planck Institute for Human Development, Berlin, Germany; Simon J. Handley and Michael F. Verde, Cognition Institute, School of Psychology, Plymouth University; Kinga Morsanyi, School of Psychology, Queen's University Belfast.

Simon J. Handley is now at Macquarie University, Faculty of Human Sciences.

We thank Junyi Dai, David Kellen, and Karl Cristoph Klauer for critical comments and helpful suggestions on the implementation of the SDT model.

Correspondence concerning this article should be addressed to Dries Trippas, Center for Adaptive Rationality, Max Planck Institute for Human Development, Lentzeallee 94, 14195 Berlin, Germany. E-mail: trippas@ mpib-berlin.mpg.de processes, respectively, and holds that the two types of processes differ qualitatively. It has proven surprisingly difficult to reach a consensus on what the critical differentiating qualities are (Evans \& Stanovich, 2013a). In the present study, we illustrate this difficulty with regard to deductive reasoning. There is general agreement that the ability to deduce logically correct conclusions for novel problems is a Type II process (Evans \& Stanovich, 2013b). In the present study, we show with the use of implicit tests that this reasoning ability possesses characteristics more typically associated with intuitive, ${ }^{1}$ heuristic responding.

De Neys (2012; also Handley \& Trippas, 2015) offered several reasons for believing that reasoning may be partly intuitive. When people fail to make normatively correct judgments, implicit measures (response time, gaze duration) often reveal sensitivity to normatively critical information. When people do make normatively correct judgments, they often fail to mention critical aspects of the normative solution in their verbal protocols. These findings show that information relevant to reasoning can be activated implicitly and thus perhaps outside of awareness, characteristic of intuitive rather than deliberative responding.

Heuristics often rely on feelings of uncertain origin. For example, people tend to rate perceptually or semantically coherent stimuli as more recognizable, likable, and true (Reber \& Unkelbach, 2010). One explanation for these implicit effects is provided by fluency misattribution accounts, which suggest that feelings that arise from a variety of sources-processing fluency,

\footnotetext{
${ }^{1}$ We use "intuitive" to indicate the quality of being nondeliberative and at least partly opaque to conscious understanding or introspection. Automaticity is a possible but not necessary component of this quality.
} 
semantic activation, familiarity, positive affect-are difficult to differentiate. As a result, these feelings are often misattributed to other sources (Reber, Winkielman, \& Schwarz, 1998; Topolinski, 2011; Whittlesea, 1993; Winkielman \& Cacioppo, 2001). Morsanyi and Handley (2012) used the fluency misattribution paradigm to investigate the possibility that deductive reasoning possesses elements that are intuitive and automatic. They hypothesized that if this were true, logical arguments should give rise to feelings of conceptual fluency even when the task does not explicitly call for reasoning. In the study, participants viewed sentences and were asked how much they liked them. Sentences that followed logically from the preceding sentences were rated as more likable. Presumably the enhanced fluency of logically valid sentences was misattributed to positive affect.

Morsanyi and Handley considered the alternative explanation that liking ratings were simply used by participants as a stand-in for validity judgments. People may have claimed to "like" a statement because they reasoned that it was logically valid. In another version of the task, participants were shown the same sentences but this time judged their logical validity. Dissociations between validity and liking judgments revealed that participants were not treating the two types of judgments as identical. Instructions that encouraged careful reasoning improved the accuracy of validity judgments but had little effect on liking. Syllogistic figure and working memory capacity, both of which are associated with reasoning effectiveness, affected validity judgments but not liking. Conversely, affective priming and a manipulation that allowed participants to discount fluency cues affected liking but not validity judgments. These double dissociations suggest that there is a component of the reasoning process that occurs implicitly even in the absence of instructions to reason. It produces feelings that have a seemingly unconscious influence on judgments unrelated to logical validity. It seems resistant to factors that are known to affect Type II processing. In sum, this component of reasoning has characteristics of nondeliberative, intuitive, heuristic responding.

A problem with Morsanyi and Handley's (2012) study was raised by Klauer and Singmann (2013; see also, Singmann, Klauer, \& Kellen, 2014), who noted that in a subset of Morsanyi and Handley's materials involving complex syllogisms (Experiments 2 and 4), logical validity was confounded with the surface content of the sentences. Klauer and Singmann found that when surface context was properly counterbalanced, they did not observe an effect of validity on the liking judgements. It is worth noting that the simpler syllogisms used by Morsanyi and Handley did not suffer from counterbalancing issues. Nevertheless, given the novelty and potentially controversial nature of their conclusions, replication and extension of their findings is warranted. In the present study we focused exclusively on relatively simple reasoning problems where the effects of implicit reasoning should most plausibly arise. We used a new set of materials that addressed the issues raised by Klauer and Singmann through randomly assigning problem contents to argument structures for each participant individually. Our aim was to demonstrate that logical validity can have an incidental influence on judgments unrelated to logic. Experiment 1 replicated the findings of Morsanyi and Handley (2012) with ratings of liking. Experiments 2 and 3 extended the results to a task further divorced from logic, judgments of physical brightness.

\section{Experiment 1}

Experiment 1 examined feelings of liking for sentences that either did, or did not follow logically from previous sentences. In addition to rigorously controlling the surface content of the materials, we also introduced a wider range of argument structures (conditionals and disjunctions in addition to syllogisms) to test the generality of Morsanyi and Handley's (2012) findings. In addition to validity, the believability of the statements was also manipulated. Believability is a factor known to drive heuristic responding in explicit reasoning tasks (Evans, Barston, \& Pollard, 1983). Believability can also be thought of as a form of semantic coherence, a factor known to produce positive affect. It is thought that feelings of semantic fluency that result from processing coherent materials are readily misattributed to feelings of liking (Topolinski \& Strack, 2009; Whittlesea, 1993). Believability is therefore predicted to increase liking. This is of interest because the implicit effect of believability, a factor associated with heuristic responding in deduction, can be compared to the implicit effect of logical validity, a factor associated with deliberate analytical responding. Also, because the mechanism of fluency misattribution underlies the predicted effects of both believability and validity, the effect of one should parallel the effect of the other.

\section{Method}

Ethics statement. All experiments were approved by the ethical committee of the Science and Environment Faculty at Plymouth University.

Participants. Forty-two psychology undergraduates from Plymouth University (6 males) participated in exchange for course credit (age range $=18-35, M=21)$.

Design. Logical validity (valid vs. invalid), argument believability (believable vs. unbelievable; note that for the conditionals this refers to the believability of the conditional statement, whereas for the other argument forms this refers to the conclusion), and problem type (conditionals vs. disjunctions vs. syllogisms) were manipulated within subjects.

Materials. We created a unique stimulus list of 144 trials for each participant: 48 conditionals ( 24 modus ponens and 24 modus tollens), 48 disjunctions (24 affirmation and 24 denial), and 48 simple syllogisms. Half of the modus ponens conditionals were valid (if $p$ then $q, p$, therefore $q$ ) and half were invalid (if $p$ then $q$, $\mathrm{p}$, therefore not $\mathrm{q}$-all negations were implicit). Half of the modus tollens conditionals were valid (if $p$ then $q$, not $q$, therefore not $p$ ) and half were invalid (if $p$ then $q$, not $q$, therefore $p$ ). Note that for the conditionals, half the valid conclusions were affirmations and half were implicit negations, and vice versa for the invalid ones. Half of the (exclusive) affirmative disjunctions were valid (50\%: $\mathrm{p}$ or $\mathrm{q}, \mathrm{p}$, therefore not $\mathrm{q} ; 50 \%$ : $\mathrm{p}$ or $\mathrm{q}, \mathrm{q}$, therefore not $\mathrm{p}$-all negations were explicit) and half were invalid (50\%: $\mathrm{p}$ or $\mathrm{q}, \mathrm{p}$ therefore q; 50\%: $\mathrm{p}$ or $\mathrm{q}, \mathrm{q}$ therefore $\mathrm{p}$ ). Half of the denial disjunctions were valid (50\%: $\mathrm{p}$ or $\mathrm{q}$, not $\mathrm{p}$, therefore $\mathrm{q} ; 50 \%$ : $\mathrm{p}$ or $\mathrm{q}, \operatorname{not} \mathrm{q}$, therefore $\mathrm{p}$ ) and half were invalid (50\%: $\mathrm{p}$ or $\mathrm{q}$, not $\mathrm{p}$, therefore not q; $50 \%$ : $\mathrm{p}$ or q, not q, therefore not $\mathrm{p}$ ). Finally, half of the syllogisms were determinately valid and half were determinately invalid (these syllogisms were also used by Trippas, Handley, \& Verde, 2013, Experiment 1 - simple condition). Believability was manipulated by randomly assigning problem contents to argument structures for each participant (Klauer \& Singmann, 
2013; Trippas et al., 2013). For the conditionals and the disjunctions, the item contents were further developed from those used by Handley, Newstead, and Trippas (2011). Examples of each of the problem types can be found in Table 1 . The full set of generating contents can be found in the Online Supplementary Materials.

Klauer and Singmann (2013) demonstrated that it is crucial for item contents and logical structures to be randomized for each participant individually. To avoid issues associated with suboptimal randomization, and to preempt other potential heuristic confounds, we used the following extensive randomization procedure. Within the conditionals 24 item contents were randomly assigned to one of the four possible modus ponens (MP) problem cells (i.e., valid-believable, invalid-believable, valid-unbelievable, invalid-unbelievable) and one of the four possible modus tollens (MT) problem cells for each participant individually for a total of 48 trials. This was done in such a way that there was no systematic link between the probability of an item content belonging to a certain cell (e.g., valid-believable) in its MP guise and it belonging to another cell (e.g., invalidbelievable) in its MT guise. The same held true within the disjunctions, with the added constraint that $\mathrm{p}$ and $\mathrm{q}$ were switched around randomly half the time to ensure the factual class was not consistently presented first or second (e.g., for half the trials the premise would be "Either the sky is green or it is blue" rather than "Either the sky is blue or it is green"). For the syllogisms we randomly assigned for each participant half of 48 problem contents to 12 valid syllogisms (each presented twice) and the remaining half of the contents to 10 invalid syllogisms (each presented twice) and 4 additional invalid syllogisms (each presented once). For half the syllogisms the conclusion direction went from $\mathrm{A}$ to $\mathrm{C}$ and for the other half from $\mathrm{C}$ to $\mathrm{A}$, and this was true for both valid and invalid syllogisms. Similarly, half the time the conclusion featured the "some" quantifier and half the time it featured the "no" quantifier, and this was the case within each validity by believability cell. Premise believability was controlled for using pseudoword nonsense middle-terms (e.g., mips). Conclusion believability was manipulated using category membership problem contents, with 16 categories (amphibians, birds, boats, cars, criminals, furniture, dogs, drinks, fish, fruits, insects, reptiles, tools, trees, vegetables, weapons) and three members per category (e.g., for amphibians: frogs, salamanders, toads; etc.). A full overview of the materials can be found in the online supplement.

Procedure. Participants were tested individually on computers running E-Prime in small groups of no more than 5. After providing informed consent, the participants were presented with the following instructions:

In this experiment we are interested in how much you like various statements.

You will repeatedly be presented with three sentences in succession for a short amount of time. Please read these sentences and indicate how much you like the final sentence on a scale from 1 (dislike it very much) to 6 (like it very much).

$$
\begin{aligned}
& 1=\text { dislike it very much } \\
& 2=\text { dislike it quite a bit } \\
& 3=\text { dislike it somewhat } \\
& 4=\text { like it somewhat } \\
& 5=\text { like it quite a bit } \\
& 6=\text { like it very much }
\end{aligned}
$$

\begin{tabular}{|c|c|c|c|c|}
\hline \multirow[b]{2}{*}{ Problem type } & \multicolumn{2}{|c|}{ Valid } & \multicolumn{2}{|c|}{ Invalid } \\
\hline & Believable & Unbelievable & Believable & Unbelievable \\
\hline \multicolumn{5}{|l|}{ Conditionals } \\
\hline \multirow[t]{3}{*}{ Modus ponens } & $\begin{array}{l}\text { P1: If a child is crying [p], } \\
\text { then it is sad [q] }\end{array}$ & $\begin{array}{l}\text { P1: If a child is crying [p], } \\
\text { then it is happy [q] }\end{array}$ & $\begin{array}{l}\text { P1: If a child is crying [p], } \\
\text { then it is happy [q] }\end{array}$ & $\begin{array}{l}\text { P1: If a child is crying [p], } \\
\text { then it is sad [q] }\end{array}$ \\
\hline & P2: A child is crying $[\mathrm{p}]$ & P2: A child is crying $[\mathrm{p}]$ & P2: A child is crying $[\mathrm{p}]$ & P2: A child is crying $[\mathrm{p}]$ \\
\hline & C: The child is sad [q] & C: The child is happy [q] & C: The child is sad $[\neg q]$ & C: The child is happy $[\neg q]$ \\
\hline \multirow[t]{3}{*}{ Modus tollens } & $\begin{array}{l}\text { P1: If a child is crying [p], } \\
\text { then it is sad [q] }\end{array}$ & $\begin{array}{l}\text { P1: If a child is laughing [p], } \\
\text { then it is sad [q] }\end{array}$ & $\begin{array}{l}\text { P1: If a child is laughing [p], } \\
\text { then it is sad [q] }\end{array}$ & $\begin{array}{l}\text { P1: If a child is crying [p], } \\
\text { then it is sad [q] }\end{array}$ \\
\hline & P2: A child is happy $[\neg q]$ & P2: A child is happy $[\neg q]$ & P2: A child is happy $[\neg q]$ & P2: A child is happy $[\neg q]$ \\
\hline & C: The child is laughing $[\neg \mathrm{p}]$ & C: The child is crying $[\neg \mathrm{p}]$ & $\mathrm{C}$ : The child is laughing [p] & C: The child is crying [p] \\
\hline \multicolumn{5}{|l|}{ Disjunctions } \\
\hline \multirow[t]{3}{*}{ Affirmation } & $\begin{array}{l}\text { P1: Either the sky is blue }[\mathrm{p}] \\
\text { or it is green }[\mathrm{q}]\end{array}$ & $\begin{array}{l}\text { P1: Either the sky is blue }[\mathrm{p}] \\
\text { or it is green }[\mathrm{q}]\end{array}$ & $\begin{array}{l}\text { P1: Either the sky is blue }[\mathrm{p}] \\
\text { or it is green }[\mathrm{q}]\end{array}$ & $\begin{array}{l}\text { P1: Either the sky is blue }[\mathrm{p}] \\
\text { or it is green }[\mathrm{q}]\end{array}$ \\
\hline & $\mathrm{P} 2:$ The sky is blue $[\mathrm{p}]$ & $\mathrm{P} 2:$ The sky is green $[\mathrm{q}]$ & P2: The sky is green [q] & P2: The sky is blue [p] \\
\hline & C: The sky is not green $[\neg q]$ & C: The sky is not blue $[\neg \mathrm{p}]$ & C: The sky is blue [p] & $\mathrm{C}$ : The sky is green $[\mathrm{q}]$ \\
\hline \multirow[t]{3}{*}{ Denial } & $\begin{array}{l}\text { P1: Either the sky is blue [p] } \\
\text { or it is green [q] }\end{array}$ & $\begin{array}{l}\text { P1: Either the sky is blue [p] } \\
\text { or it is green }[\mathrm{q}]\end{array}$ & $\begin{array}{l}\text { P1: Either the sky is blue [p] } \\
\text { or it is green }[\mathrm{q}]\end{array}$ & $\begin{array}{l}\text { P1: Either the sky is blue [p] } \\
\text { or it is green [q] }\end{array}$ \\
\hline & P2: The sky is not green $[\neg q]$ & P2: The sky is not blue $[\neg \mathrm{q}]$ & P2: The sky is not blue $[\neg \mathrm{p}]$ & P2: The sky is not green $[\neg \mathrm{q}]$ \\
\hline & C: The sky is blue [p] & C: The sky is green $[\mathrm{p}]$ & C: The sky is not green $[\neg q]$ & C: The sky is not blue $[\neg \mathrm{p}]$ \\
\hline \multirow[t]{3}{*}{ Syllogisms } & P1: All wines are mips & P1: All wines are mips & P1: All wines are mips & P1: All wines are mips \\
\hline & P2: No mips are tools & $\mathrm{P} 2$ : No mips are drinks & P2: No mips are drinks & P2: No mips are tools \\
\hline & C: No wines are tools & C: No wines are drinks & C: Some wines are drinks & C: Some wines are tools \\
\hline
\end{tabular}

Table 1

Examples of the Materials Used in Experiments 1-3

Note. $\mathrm{p}$ and $\mathrm{q}$ indicate the abstract structure of the arguments. $\neg \mathrm{p}$ and $\neg \mathrm{q}$ stand for "not p" and "not q", respectively. 
When you make the liking judgment, please focus on your feeling about the statement. Do not think about why you like or dislike the statement, just go with your intuition and gut-feelings.

Please try to use the full extent of the scale (so use all possible values from 1-6).

For each problem trial, participants were first presented with the major premise for 2 seconds, then with the minor premise for 2 seconds, and finally with the conclusion and the response scale. After making a response, the participant was instructed to press space to advance to the next problem.

\section{Results}

Analysis approach. We used a top-down Bayesian analysis of variance (ANOVA) hypothesis testing approach with default priors (Rouder, Morey, Speckman, \& Province, 2012) followed up with a Bayesian hierarchical unequal variance signal detection theory (SDT, see, e.g., Macmillan \& Creelman, 2005) estimation approach (Kruschke, 2014; Lee \& Wagenmakers, 2013). ${ }^{2}$ One advantage of a Bayesian hypothesis testing approach over its frequentist alternative is that the former allows us to interpret data as providing evidence for the null by calculating Bayes factors (BFs). After identifying the main effects and interactions which had sufficient support given the data, indices of sensitivity were estimated for each factor using a Bayesian hierarchical unequal variance SDT model (Morey, Pratte, \& Rouder, 2008). Here, sensitivity refers to differences in the distribution of liking ratings between levels of a factor, for example, between logically valid and invalid statements. The SDT analysis is a useful supplement in that it considers the whole distribution of ratings but makes no assumptions about the uniformity of the distributions, or the equality of their variance ratio (Heit \& Rotello, 2014; Trippas, Handley, \& Verde, 2014).

ANOVA. We analyzed the liking judgments using a 2 (Logical Validity: valid vs. invalid) $\times 2$ (Argument Believability: believable vs. unbelievable) $\times 3$ (Problem Type: conditional vs. disjunction vs. syllogism) top down BF ANOVA with default JZS priors and a random effect for participants to account for the repeated measures nature of the three factors. ${ }^{3}$ BFs were calculated by comparing the full model containing all main effects and interactions with the model in which the effect of interest was removed. $\mathrm{BF}>1$ indicate more evidence in favor of the inclusion of the effect, $\mathrm{BF}<1$ indicate more evidence against the inclusion of the effect.

Logically valid conclusions were liked more than logically invalid ones, $\mathrm{BF}=5.8 * 10^{165}$. Believable arguments were also liked more than unbelievable ones, $\mathrm{BF}=1.5^{*} 10^{193}$. There was also a main effect of problem type, suggesting certain argument forms were liked more than others, $\mathrm{BF}=366$. Logical validity and problem type interacted, suggesting the effect of logic on liking differed as a function of argument form, $\mathrm{BF}=2.6^{*} 10^{12}$. There was evidence against the logic by belief interaction $(\mathrm{BF}=0.26)$ and the three-way interaction $(\mathrm{BF}=0.33)$. The data was uninformative with regards to the Belief $\times$ Problem Type interaction $(\mathrm{BF}=0.98)$. Raw means and standard deviations for the liking judgments per condition can be found in Table 2 .

SDT estimation. We estimated sensitivity to each of the effects for which substantial evidence was found given the model and the data (Logic, Belief, Problem Type, and the Logic $\times$ Problem Type interaction). We estimated $d_{a}$ for each factor by applying a Bayesian hierarchical unequal variance SDT model using JAGS to the liking ratings (sensitivity estimates are based on the means of the hyper-distributions; see code in Online Supplementary Materials for specifics). We ran four chains with 20,000 iterations each, with 1,000 samples burn-in and no thinning. For all of the reported analysis, all chains converged well (all Rhat $<1.10$ ). The reported $95 \%$ highest density intervals (HDI) mark the span of values that are most credible and cover $95 \%$ of the distribution (Kruschke, 2014).

Participants liked valid problems more than invalid ones, $M$ $d_{a}$-logic $=0.89,95 \%$ HDI $=0.52-1.33$. Similarly, participants liked believable arguments more than unbelievable ones, $M$ $d_{a}$-belief $=0.85,95 \%$ HDI $=0.59-1.14$. Participants did not credibly like conditionals more than disjunctions, $M d_{a}-\mathrm{PT}-\mathrm{CD}=$ $0.03,95 \% \mathrm{HDI}=-0.09-0.15$. In contrast, there was a preference for conditionals over syllogisms, $M d_{a}$-PT-CS $=0.16,95 \%$ HDI $=0.04-0.28$. There was a marginal trend for disjunctions to be preferred over syllogisms, $M d_{a}$-PT-DS $=0.20,95 \%$ HDI $=-0.05-0.51$. We also investigated the Logic $\times$ Problem Type interaction by estimating sensitivity to logic for each problem type individually. Participants liked valid conditionals more than invalid ones, $M d_{a}$-logic-conditional $=0.81,95 \%$ HDI $=$ 0.58-1.06. Participants also liked valid disjunctions more than invalid ones, $M d_{a}$-logic-disjunction $=1.37$, 95\% HDI $=0.69-$ 2.13. Participants liked valid syllogisms more than invalid ones, $M$ $d_{a}$-logic-syllogism $=0.53,95 \%$ HDI $=0.27-0.83$.

\section{Discussion}

Although the task made no reference to logical validity, participants liked statements more when they formed part of a logically valid argument. The size of the validity effect was comparable to that of believability, which also enhanced liking. Much previous work has shown that the believability of a statement can influence explicit judgments of logical validity. According to dual process theories of deduction, belief bias (Evans et al., 1983) occurs when the automatic activation of semantic information encourages heuristic responding, a Type I process. Because the influence of existing beliefs is intuitive, it may not be surprising that believability would have an implicit effect on unrelated judgments. Indeed, other factors related to semantic coherence have been shown to implicitly influence feelings of liking (Topolinski \& Strack, 2009; Whittlesea, 1993). On the other hand, it seems uncharacteristic for deliberative reasoning, a Type II process, to have a similar implicit effect on unrelated judgments.

Morsanyi and Handley (2012), whose findings are conceptually replicated in Experiment 1, proposed that people are sensitive to logical structure at an intuitive level. We will examine the case for this in detail later. For the moment, we consider an alternative possibility. It may be that participants, uninstructed, decided to use

\footnotetext{
${ }^{2}$ In all three experiments, the use of traditional frequentist and alternative Bayesian analyses produced the same conclusions. These alternative analyses can be found in the online supplementary materials.

${ }^{3}$ We used the anovaBF() function from the BayesFactor $\mathrm{R}$ package with settings rscaleFixed = "medium" and whichModel = "top". The exact syntax can be found in the online supplementary materials. JAGS code for the SDT model can also be found in the online supplement.
} 
Table 2

Experiment 1: Liking Judgements Per Condition

\begin{tabular}{llccllcr}
\hline & \multicolumn{3}{c}{ Valid, $M(S D)$} & & \multicolumn{3}{c}{ Invalid, $M(S D)$} \\
\cline { 2 - 3 } Problem type & Believable & Unbelievable & Total & & Believable & Unbelievable & Total \\
\hline Conditional & $4.67(1.48)$ & $3.51(1.73)$ & $4.09(1.71)$ & & $3.32(1.72)$ & $2.32(1.49)$ & $2.82(1.68)$ \\
Disjunction & $4.96(1.33)$ & $3.35(1.87)$ & $4.16(1.81)$ & & $3.28(1.79)$ & $2.10(1.39)$ & $2.69(1.71)$ \\
Syllogism & $4.16(1.51)$ & $2.99(1.61)$ & $3.58(1.67)$ & & $3.54(1.63)$ & $2.24(1.27)$ & $2.89(1.60)$ \\
\hline
\end{tabular}

normative validity as a criterion for liking. As noted earlier, Morsanyi and Handley offered evidence against this possibility with the observation that factors mediating the validity effect in an explicit reasoning task differed from those mediating the validity effect in the implicit liking task. In other words, participants were not treating the two types of judgments as identical. Nevertheless, it seems plausible that if participants were to intentionally deduce the validity of the statements, they might view a state of "correctness" as more likable. The next experiment aimed to avoid this by using an implicit task with no plausible connection to logical validity.

\section{Experiment 2}

Manipulating the ease of perceptual processing can influence unrelated judgments. For example, enhanced picture clarity leads to higher ratings of liking (Reber, Winkielman, \& Schwarz, 1998), and perceptual priming and text uniformity lead to increased familiarity judgments (Jacoby \& Whitehouse, 1989; Whittlesea \& Leboe, 2003). According to the fluency misattribution account, perceptual fluency produces a feeling that is ambiguous and difficult to distinguish from other internal signals (e.g., Mandler, Nakamura, \& Van Zandt, 1987). The corollary is that internal signals produced by other processes might be misattributed to perceptual processing. We hypothesized that this would be the case for the implicit aspects of logical validity observed in Experiment 1. In Experiment 2, participants viewed the materials from the previous experiment but this time judged the physical brightness of the statements as presented on the screen. To the layman, the criteria for brightness are more narrowly defined than for "liking" and do not plausibly include the semantic or syntactic content of the materials. To ensure an explicit focus on physical cues, brightness of the stimuli was varied independently of the other variables (i.e., logical validity and argument believability), and participants were shown examples of low and high brightness sentences to ensure an understanding of what constituted a correct response within the context of the experiment. Given the observed positive relationship between enhanced perceptual fluency and positive affect (Reber et al., 1998; Winkielman \& Cacioppo, 2001), the findings of Experiment 1 lead to the prediction that validity (and believability) should encourage higher ratings of physical brightness.

\section{Method}

Participants. Forty-eight psychology undergraduates from Plymouth University (6 male) participated in exchange for course credit (age range $=18-41, M=21$ ).
Design. Logical validity (valid vs. invalid), argument believability (believable vs. unbelievable), brightness (high vs. low), and problem type (conditionals vs. disjunctions vs. syllogisms) were manipulated within subjects.

Materials. Materials were created as in Experiment 1. Brightness was manipulated by randomly assigning half of the problems in each Logic $\times$ Belief $\times$ Problem Type cell to be high or low brightness. High and low brightness trials differed in the contrast between the problem text (shades of gray) and the background (white). All of the text presented within a trial (including the major premise, the minor premise, the conclusion, and the response scale) had identical contrast levels. Stimuli were created by assigning a red-green-blue (RGB) value $(x, x, x)$, with $x$ drawn from a normal distribution with $\mu=30, \sigma=1$ for high, and $\mu=40$, $\sigma=1$ for low brightness. Based on pilot work, these values ensured that brightness judgments were possible without being trivially easy.

Procedure. Participants were tested individually on computers under controlled lighting conditions in small groups of no more than five. After providing informed consent, the participants were presented with examples of high and low brightness sentences (see Figure 1). The following instructions were presented:

You will repeatedly be presented with three sentences in succession for a short amount of time. Please read these sentences and indicate whether the final statement is high or low brightness on a scale from 1 (certainly low brightness) to 6 (certainly high brightness).

$1=$ certainly low brightness

$2=$ probably low brightness

In this experiment we are interested in brightness judgements.

This is a high brightness sentence

This is a low brightness sentence

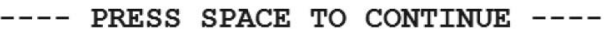

Figure 1. Screenshot from the experiment. High brightness sentences had red-green-blue (RGB) values around (30, 30, 30), low brightness sentences had RGB values around $(40,40,40)$. All sentences were presented against a white $(255,255,255)$ background. 
Table 3

Experiment 2: Brightness Judgments Per Condition

\begin{tabular}{|c|c|c|c|c|c|c|}
\hline \multirow[b]{2}{*}{ Problem type } & \multicolumn{2}{|c|}{ Logic, $M(S D)$} & \multicolumn{2}{|c|}{ Belief, $M(S D)$} & \multicolumn{2}{|c|}{ Brightness, $M(S D)$} \\
\hline & Valid & Invalid & Believable & Unbelievable & High & Low \\
\hline Conditional & $3.96(1.46)$ & $3.71(1.47)$ & $3.99(1.45)$ & $3.68(1.48)$ & $4.18(1.44)$ & $3.49(1.41)$ \\
\hline Disjunction & $4.01(1.46)$ & $3.64(1.52)$ & $3.94(1.48)$ & $3.72(1.52)$ & $4.14(1.50)$ & $3.52(1.45)$ \\
\hline Syllogism & $3.88(1.43)$ & 3.69 (1.49) & $3.87(1.43)$ & $3.70(1.48)$ & $4.13(1.44)$ & 3.43 (1.39) \\
\hline Total & $3.95(1.45)$ & 3.68 (1.49) & $3.93(1.45)$ & $3.70(1.49)$ & $4.15(1.46)$ & $3.48(1.42)$ \\
\hline
\end{tabular}

$3=$ guess low brightness

$4=$ guess high brightness

$5=$ probably high brightness

6 = certainly high brightness

The brightness differences are quite subtle, so make use of your gut feeling and intuition when making a judgment. Occasionally you will have to guess. Once again, make sure you do this on the basis of your gut feeling.

Also make sure you use the full extent of the scale (so use all possible values from 1 to 6 ).

There will be a short memory test at the end of the experiment to check whether you read all the sentences, so make sure that you read all of them.

\section{Results}

ANOVA. We analyzed the brightness judgments using a 2 (Logical Validity: valid vs. invalid) $\times 2$ (Argument Believability: believable vs. unbelievable) $\times 2$ (Brightness: high contrast vs. low contrast) $\times 3$ (Problem Type: conditional vs. disjunction vs. syllogism) top down BF ANOVA with default JZS priors and a random effect for participants as in Experiment 1. Logically valid conclusions were rated as more bright than invalid ones, $\mathrm{BF}=$ $1.3^{*} 10^{13}$. Believable arguments were also rated as more bright than unbelievable ones, $\mathrm{BF}=2.8 * 10^{9}$. High contrast conclusions were judged as brighter than low contrast conclusions, $\mathrm{BF}=9.0$ * $10^{84}$. There was substantial evidence against inclusion of the remaining effects (all $\mathrm{BF}<0.13$ ). Raw means and standard deviations are presented in Table 4.

SDT estimation. We estimated sensitivity to each of the components in favor of which substantial evidence was found (logic, belief, brightness) using a Bayesian hierarchical UVSDT model. Participants judged valid arguments as physically brighter than invalid arguments, $M \mathrm{~d}_{\mathrm{a}}$-logic $=0.24,95 \%$ HDI $=0.04-0.46$. Participants judged believable arguments as brighter than unbelievable arguments, $M \mathrm{~d}_{\mathrm{a}}$-belief $=0.21,95 \% \mathrm{HDI}=0.03-0.41$. As would be expected, participants judged high brightness problems as brighter than low brightness problems, $M \mathrm{~d}_{\mathrm{a}}$-brightness $=$ $0.68,95 \%$ HDI $=0.34-1.12$.

\section{Discussion}

Participants judged valid statements which followed logically from the preceding sentences as being physically brighter than invalid statements. As in the previous experiment, there was a parallel effect of believability. Believable statements were judged to be brighter than unbelievable statements. The implicit influence of logical validity on a judgment whose criteria involved purely physical characteristics is surprising. However, the results are conceptually consistent with those of Experiment 1 and extend them to a task that is even further removed from the explicit requirement to reason logically. Experiment 3 sought to replicate the brightness findings alongside a manipulation of affect that, like believability, was predicted to influence brightness judgments.

\section{Experiment 3}

Theories of embodied emotion hold that adopting a facial expression is sufficient to produce its associated affective state. Because people are typically unclear about the source of the affect, it is readily misattributed to an external source. For example, Strack, Martin, and Stepper (1988) found that people rated cartoons as funnier when they held a pen between their teeth (facilitating muscles responsible for smiling) than when they held it between their lips. Verde, Stone, Hatch, and Schnall (2010) found that the same manipulation of expression led people to judge words as more familiar.

In Experiments 1 and 2, the manipulation of believability served to demonstrate that a factor known to drive heuristic responding

Table 4

Experiment 3: Brightness Judgments Per Condition

\begin{tabular}{|c|c|c|c|c|c|c|c|c|}
\hline \multirow[b]{2}{*}{ Problem type } & \multicolumn{2}{|c|}{ Logic, $M(S D)$} & \multicolumn{2}{|c|}{ Belief, $M(S D)$} & \multicolumn{2}{|c|}{ Brightness, $M(S D)$} & \multicolumn{2}{|c|}{ Pen condition, $M(S D)$} \\
\hline & Val & Inval & Bel & Unbel & High & Low & $\mathrm{Fac}$ & Inh \\
\hline Condition & $4.02(1.49)$ & $3.84(1.58)$ & $3.98(1.52)$ & $3.89(1.56)$ & $4.19(1.51)$ & $3.68(1.52)$ & $3.97(1.57)$ & $3.90(1.50)$ \\
\hline Disjunction & $4.00(1.56)$ & $3.69(1.58)$ & $3.95(1.56)$ & $3.74(1.59)$ & $4.11(1.56)$ & $3.58(1.55)$ & $3.91(1.60)$ & $3.78(1.55)$ \\
\hline Syllogism & $3.99(1.50)$ & $3.76(1.53)$ & $3.93(1.49)$ & $3.81(1.54)$ & $4.14(1.51)$ & $3.61(1.48)$ & $3.97(1.55)$ & $3.78(1.49)$ \\
\hline Total & $4.00(1.56)$ & $3.76(1.52)$ & $3.95(1.52)$ & $3.81(1.57)$ & $4.14(1.53)$ & $3.62(1.52)$ & $3.95(1.57)$ & $3.82(1.52)$ \\
\hline
\end{tabular}

Note. $\quad$ Val = valid; Inval = invalid; Bel = believable; Unbel = unbelievable; Fac $=$ facilitation; Inh $=$ inhibition. 
can implicitly influence liking and brightness judgments. The parallel effects of believability and logical validity suggest that like the former, the latter might stem from a process possessing heuristic, intuitive qualities. In Experiment 3, the manipulation of facial expression was meant to serve a similar function. The misattribution of affective feelings produced by facial expression is believed to result from intuitive heuristics that people use to interpret ambiguous internal signals. Finding parallel effects on brightness ratings of expression and logical validity might be suggestive about the qualities of the process responsible for the latter effect.

\section{Method}

Participants. Seventy-four participants sampled from the population in Plymouth participated in exchange for a small fee (age range $=18-70, M=31$ ).

Design. Logical validity (valid vs. invalid), argument believability (believable vs. unbelievable), brightness (high vs. low), and argument type (conditionals vs. disjunctions vs. syllogisms) were manipulated within subjects. Simulated smiling (smile-facilitation vs. smile-inhibition) was manipulated between subjects. Participants were randomly assigned to the facilitation $(N=37)$ or the inhibition $(N=37)$ condition. One person dropped out during the experiment because they struggled with the brightness judgment task.

Procedure. The procedure was identical to that of Experiment 2, with the exception that people were asked to put a pen between either their lips or their teeth for the duration of the experiment. The experimenter demonstrated to each participant exactly how to put the pen in their mouth. Participants were allowed breaks between trials but were instructed to put the pen back in the correct position upon continuing. The experimenter monitored pen position throughout the study.

\section{Results}

ANOVA. We analyzed the brightness judgments using a 2 (Logical Validity: valid vs. invalid) $\times 2$ (Argument Believability: believable vs. unbelievable) $\times 2$ (Brightness: high contrast vs. low contrast) $\times 3$ (Problem Type: conditional vs. disjunction vs. syllogism) $\times 2$ (Simulated Smiling: smile-facilitation vs. smileinhibition) top-down BF ANOVA with default JZS priors and a random effect for participants to account for the mixed nature of the design. Logically valid conclusions were rated as brighter than logically invalid ones, $\mathrm{BF}=3.6^{*} 10^{15}$. Believable arguments were rated as brighter than unbelievable ones, $\mathrm{BF}=7720$. High contrast sentences were rated as brighter than low contrast ones, $\mathrm{BF}=3.0$ * $10^{77}$. With the exception of the Simulated Smiling $\times$ Brightness interaction $(\mathrm{BF}=1.4)$ and the Simulated Smiling $\times$ Belief interaction $(\mathrm{BF}=0.50)$, for which the data was uninformative, there was strong support in favor of the null for all remaining effects (BFs <0.12). Means and standard deviations of the brightness judgments can be found in Table 3 .

Given our a priori interest in the effect of simulated smiling and the inconclusive evidence for several interactions which included simulated smiling as a component, we investigated the manipulation in more detail. A BF contingency table analysis of independence assuming independent multinomial distributions was run to compare the distribution of brightness ratings for the simulated smiling and the simulated frowning conditions. The analysis suggested brightness ratings were distributed differently in both conditions, $\chi^{2}(5)=72.6, \mathrm{BF}=8.5^{*} 10^{8}$.

SDT estimation. Using a Bayesian hierarchical UVSDT model, we estimated sensitivity to each of the components for which strong evidence was found in the ANOVA (logic, belief, brightness). Simulated smiling was assessed using a Bayesian ordered probit regression given its between subjects nature (cf. Kruschke, 2014). Participants' judged valid arguments as more physically bright than invalid ones, $M \mathrm{~d}_{\mathrm{a}}$-logic $=0.30,95 \%$ HDI $=0.03-0.60$. There was a marginal trend for participants to judge believable arguments as brighter than unbelievable ones, $M \mathrm{~d}_{\mathrm{a}}$-belief $=0.24,95 \%$ HDI $=-0.10-0.64(77 \% \mathrm{HDI}=$ $0-.36)$. High brightness sentences were judged as brighter than low brightness sentences, $M \mathrm{~d}_{\mathrm{a}}$-brightness $=1.42,95 \%$ HDI $=$ $0.28-3.49$. Finally, a Bayesian ordered probit regression demonstrated that the effect size of simulated smiling credibly differed from $0, M d=0.11,95 \%$ HDI $=0.07-0.16$.

\section{Discussion}

Replicating the key result of Experiment 2, logically valid statements were judged to be physically brighter than their invalid counterparts. Believability and facial expression (simulated smiling) also tended to influence perceived brightness, although evidence for the former was mixed, and exclusion of the latter from the best fitting model suggests that it is a weaker effect.

\section{General Discussion}

Dual process theory has been able to accommodate a range of disparate phenomena within a common framework, one which draws converging support from other domains of cognition and neuroscience (Evans, 2008). However, the fundamental question regarding which qualities distinguish Type I and Type II processes remains a matter of debate (Evans \& Stanovich, 2013a). According to the dual process view, the computation of logical validity requires deliberation, time and cognitive control. Heuristic responses, in contrast, are intuitive, fast, and often automatic. We examined this dichotomy using implicit tests of reasoning. Participants viewed sentences containing inherent logical structure. They were not asked to reason about the sentences but instead judged simply how much they liked them (Experiment 1) or how bright they appeared on the screen (Experiments 2 and 3). Sentences that followed logically from the preceding sentences were rated as more likable and judged to be brighter than sentences that were logically invalid. The implicit sensitivity to logical structure revealed in judgments unrelated to reasoning suggests something about the nature of the reasoning process. Although ostensibly a Type II process, reasoning does not seem to be exclusively deliberative but instead possesses some qualities typically associated with intuitive, heuristic processes.

Heuristics tend to be nondeliberative because they are implemented quickly, sometimes automatically, and because people may have limited insight about the source of these heuristics. An example is the heuristic of using the processing fluency of a stimulus to infer other, unrelated properties (for an alternative example in terms of retrieval fluency, see Hertwig, Herzog, 
Schooler, \& Reimer, 2008). Lack of insight into this process is suggested by the fact that feelings of fluency can be misinterpreted in different ways depending on the context (e.g., as feelings of familiarity, liking, brightness; Mandler et al., 1987). Moreover, people are more likely to make use of the heuristic when not aware of the source of fluency. When made aware of the source, they correctly reject fluency as irrelevant to the judgment at hand (Goldinger \& Hansen, 2005; Jacoby \& Whitehouse, 1989; Schwarz \& Clore, 1983; Whittlesea, 2002). In the present study, we know that participants understood the brightness task because they were able to differentiate different levels of text-tobackground contrast with above-chance accuracy. There is no plausible reason to allow logical validity to inform judgments of physical brightness. Nevertheless, there was a validity effect. It seems reasonable to infer that participants lacked insight or awareness of the implicit effects of logical structure. Had they been aware, they should have rejected the information as irrelevant.

Along with logical validity, sentence believability and facial expression also influenced judgments of liking and brightness. The notion that participants were deliberately basing their judgments on such a disparate collection of (irrelevant) information seems implausible. An alternative explanation draws on a large body of work investigating fluency misattribution. Psychological processes produce signals that are often ambiguous and require interpretation. Other work has shown that positive affect produced by an unrelated source can be misinterpreted as perceptual clarity or familiarity (Strack et al., 1988; Verde et al., 2010), and feelings associated with conceptual coherence can be misinterpreted as feelings of liking (Topolinski \& Strack, 2009; Whittlesea, 1993). The implicit effects of facial expression and believability therefore have precedent, although our findings are novel with respect to the materials and tasks used. Aside from Morsanyi and Handley's (2012) earlier study, the implicit effect of validity is novel. We speculate that comprehending logical validity, much like comprehending conceptual cohesion, produces feelings that are ambiguous and open to misinterpretation. The implication is that the reasoning process, at some level, produces output that is opaque to understanding or introspection.

Although participants were not instructed to make logical deductions while reading the sentences, we cannot rule out the possibility that some may have done so spontaneously. This would not change the conclusions drawn so far. Whether or not reasoning was instigated deliberately, the implicit effects of reasoning seem to be nondeliberate. However, there are other issues for which the question of deliberate reasoning is relevant. Morsanyi and Handley (2012) proposed that the implicit effect of logical structure was evidence that the reasoning process itself was at least partly intuitive. They noted that the validity effects observed in the explicit and implicit reasoning tasks responded differently to various manipulations. For example, different instructions (known to influence explicit reasoning) mediated the validity effect in the explicit but not the implicit task. Conversely, fluency cues (known to influence heuristic responding) mediated the validity effect in the implicit but not the explicit task.

Although we did not have an explicit reasoning condition for direct comparison, post hoc examination of response latencies revealed a pattern atypical of explicit reasoning performance. Deliberate reasoning, being slow and effortful, is less accurate under time pressure (Evans \& Curtis-Holmes, 2005; Trippas et al.,
2013). This would lead us to expect that, if people had been reasoning deliberately, the fastest responses in the implicit task would show the weakest, or even no effect of validity. Combining data from Experiments 2 and 3, the brightness judgments were divided into faster $(M=1.5 \mathrm{~s})$ and slower $(M=3.8 \mathrm{~s})$ responses via a median split on response latencies. A 2 (Conclusion Validity: valid vs. invalid) $\times 2$ (Response Speed: faster vs. slower) $\mathrm{BF}$ ANOVA showed that there was no evidence in favor or against a logic $\mathrm{x}$ response speed interaction, $\mathrm{BF}=1.28$. Nevertheless, we conducted follow-up analyses which indicated that the effect of logic on brightness judgments was present for both the faster $(M$ $\mathrm{d}_{\mathrm{a}}$-logic-quick $=0.17,95 \%$ HDI $\left.=0.13-0.21\right)$ and slower responses $\left(M \mathrm{~d}_{\mathrm{a}}\right.$-logic-slow $=0.13,95 \%$ HDI $\left.=0.08-0.17\right)$. Contrary to what would be expected if the validity effect was based on deliberate reasoning, the effect was descriptively larger for the faster responses.

These differences lead us to believe that whatever drives sensitivity to logical validity in the implicit tasks, it is only a component of the explicit reasoning process. Explicit reasoning may be largely deliberative and resource-intensive, but it may include components that are nondeliberative and more intuitive. There is some evidence from the larger literature on reasoning models that speaks to this idea. We already noted De Neys' (2012) discussion of the finding that people appear to be intuitively sensitive to the conflict between formal norms such as probability theory and heuristic factors such as stereotypes, but often unable to articulate critical aspects of the reasoning process, such as this conflict detection. These findings led to the proposal of a "logical intuitions" model of reasoning according to which people have some degree of intuitive sensitivity to all formal norms. According to this model, the analytic-heuristic conflict is always detected, but formal responses are not made because it is not inhibited. On the other side of the spectrum resides the "default-interventionist" model of reasoning discussed by Evans and Stanovich (2013a). According to this model, heuristic considerations always precede potential analysis due to the autonomous nature of the former and the necessity for working memory involvement of the latter. Occasional failures to detect the conflict (i.e., monitoring failures) are considered one of the main culprits for heuristic responding. Other possible contenders tread the middle ground (e.g., Handley \& Trippas, 2015; Pennycook, Fugelsang, \& Koehler, 2015; Sloman, 2014; Thompson, Prowse-Turner, \& Pennycook, 2011). According to Handley and Trippas' Parallel Processing Model (PPM), for instance, intuition and deliberation act in a parallel, race-like fashion, simultaneously integrating the various problem components (e.g., structure and knowledge) that are present during the task at hand. The reason why occasionally heuristic considerations outweigh analysis and vice versa is determined by features such as the complexity of these components and the ease with which they are processed. Our demonstration of implicit sensitivity to somerelatively simple-logical structures is consistent with this latter account. Whether implicit sensitivity to more complex formal norms will also arise, as predicted by the logical intuitions model, remains to be seen (see Handley \& Trippas, 2015, for a more detailed discussion of these and several other reasoning models).

We have proposed that valid arguments are associated with increased fluency, but as of yet we can only speculate on the mechanism that brings this about. Theories of discourse comprehension such as Kintsch's (1988) construction-integration model 
suggest that readers implicitly construct situational models through associations and automatic inferences. It may be more difficult to create coherent semantic models of invalid arguments, perhaps because they describe impossible situations. Morsanyi and Handley (2012) offered an explanation along these lines for their liking judgment results. They proposed that the ease with which the concluding statement of a valid argument fits with the model constructed from earlier statements produces a sense of fluency that is then misattributed to another source. Whether fluency is tied to the ease of creating any coherent semantic model, or more specifically to the ability to create a logically coherent model, is an interesting question for future investigation. Some support for the latter comes from a study by Lea, O'Brien, Fisch, Noveck, and Braine (1990) in which participants read narratives containing propositional chains, for example:

Jerry thought: "I would like to wear either my checkered or my striped shirt. Of course, if I wear my striped shirt I'll have to wear my matching blue trousers." Jerry then discovered that his striped shirt was wrinkled.

Later, participants preferred sentences which suggested that they drew logically valid inferences (e.g., Jerry thought: "I guess I won't have to look for my blue trousers then") over sentences that were also plausible within the situational narrative but logically invalid (Jerry thought: "I guess I'd better start looking for my blue trousers"). Participants seemed to have generated the logical conclusions but were not necessarily aware of having done so.

The invalid arguments used in our study were determinately invalid, meaning that it is impossible to construct a situation model in which the conclusion holds given the premises. It would be useful to compare these with indeterminately invalid arguments for which it is possible to create a model that fits the conclusion. If fluency is linked simply to the ease of creating a coherent model, the validity effect might not be observed in the latter case whereas it would be observed in both cases if fluency is linked to logical validity. ${ }^{4}$ We should emphasize that either possibility is consistent with the proposal that sensitivity to logical structure can arise in ways that defy easy characterization as a strictly conscious, deliberative Type II process.

${ }^{4}$ We are grateful to Karl Cristoph Klauer for proposing this experiment.

\section{References}

De Neys, W. (2012). Bias and conflict: A case for logical intuitions. Perspectives on Psychological Science, 7, 28-38. http://dx.doi.org/10 $.1177 / 1745691611429354$

Evans, J. St. B. T. (2008). Dual-processing accounts of reasoning, judgment, and social cognition. Annual Review of Psychology, 59, 255-278. http://dx.doi.org/10.1146/annurev.psych.59.103006.093629

Evans, J. St. B. T., Barston, J. L., \& Pollard, P. (1983). On the conflict between logic and belief in syllogistic reasoning. Memory \& Cognition, 11, 295-306. http://dx.doi.org/10.3758/BF03196976

Evans, J. St. B. T., \& Curtis-Holmes, J. (2005). Rapid responding increases belief bias: Evidence for the dual process theory of reasoning. Thinking \& Reasoning, 11, 382-389. http://dx.doi.org/10.1080/135467805 42000005

Evans, J. St. B. T., \& Stanovich, K. E. (2013a). Dual-process theories of higher cognition: Advancing the debate. Perspectives on Psychological Science, 8, 223-241. http://dx.doi.org/10.1177/1745691612460685
Evans, J. St. B. T., \& Stanovich, K. E. (2013b). Theory and metatheory in the study of dual processing: Reply to comments. Perspectives on Psychological Science, 8, 263-271. http://dx.doi.org/10.1177/ 1745691613483774

Gigerenzer, G., Todd, P. M., \& the ABC Research Group. (1999). Simple heuristics that make us smart. New York, NY: Oxford University Press.

Goldinger, S. D., \& Hansen, W. A. (2005). Remembering by the seat of your pants. Psychological Science, 16, 525-529. http://dx.doi.org/10 .1111/j.0956-7976.2005.01569.x

Handley, S. J., Newstead, S. E., \& Trippas, D. (2011). Logic, beliefs, and instruction: A test of the default interventionist account of belief bias. Journal of Experimental Psychology: Learning, Memory, and Cognition, 37, 28-43. http://dx.doi.org/10.1037/a0021098

Handley, S. J., \& Trippas, D. (2015). Dual processes, knowledge, and structure: A critical evaluation of the default interventionist account of biases in reasoning and judgement. Psychology of Learning and Motivation, 62, 33-56.

Heit, E., \& Rotello, C. M. (2014). Traditional difference-score analyses of reasoning are flawed. Cognition, 131, 75-91. http://dx.doi.org/10.1016/ j.cognition.2013.12.003

Hertwig, R., Herzog, S. M., Schooler, L. J., \& Reimer, T. (2008). Fluency heuristic: A model of how the mind exploits a by-product of information retrieval. Journal of Experimental Psychology: Learning, Memory, and Cognition, 34, 1191-1206. http://dx.doi.org/10.1037/a0013025

Jacoby, L. L., \& Whitehouse, K. (1989). An illusion of memory: False recognition influenced by unconscious perception. Journal of Experimental Psychology: General, 118, 126-135. http://dx.doi.org/10.1037/ 0096-3445.118.2.126

Kahneman, D. (2011). Thinking, fast and slow. New York, NY: Farrar, Straus \& Giroux.

Kintsch, W. (1988). The role of knowledge in discourse comprehension: A construction-integration model. Psychological Review, 95, 163-182. http://dx.doi.org/10.1037/0033-295X.95.2.163

Klauer, K. C., \& Singmann, H. (2013). Does logic feel good? Testing for intuitive detection of logicality in syllogistic reasoning. Journal of Experimental Psychology: Learning, Memory, and Cognition, 39, 12651273. http://dx.doi.org/10.1037/a0030530

Kruschke, J. (2014). Doing Bayesian data analysis: A tutorial with $R$ and BUGS (2nd ed.). Amsterdam, the Netherlands: Elsevier.

Lea, R. B., O'Brien, D. P., Fisch, S. M., Noveck, I. A., \& Braine, M. D. (1990). Predicting propositional logic inferences in text comprehension. Journal of Memory and Language, 29, 361-387. http://dx.doi.org/10 .1016/0749-596X(90)90005-K

Lee, M. D., \& Wagenmakers, E.-J. (2013). Bayesian modeling for cognitive science: A course. New York, NY: Cambridge University Press http://dx.doi.org/10.1017/CBO9781139087759

Macmillan, N. A., \& Creelman, C. D. (2005). Detection theory: A user's guide (2nd ed.). Mahwah, NJ: Erlbaum.

Mandler, G., Nakamura, Y., \& Van Zandt, B. J. S. (1987). Nonspecific effects of exposure on stimuli that cannot be recognized. Journal of Experimental Psychology: Learning, Memory, and Cognition, 13, 646648. http://dx.doi.org/10.1037/0278-7393.13.4.646

Morey, R. D., Pratte, M. S., \& Rouder, J. N. (2008). Problematic effects of aggregation in z ROC analysis and a hierarchical modeling solution. Journal of Mathematical Psychology, 52, 376-388. http://dx.doi.org/10 .1016/j.jmp.2008.02.001

Morsanyi, K., \& Handley, S. J. (2012). Logic feels so good-I like it! Evidence for intuitive detection of logicality in syllogistic reasoning. Journal of Experimental Psychology: Learning, Memory, and Cognition, 38, 596-616. http://dx.doi.org/10.1037/a0026099

Pennycook, G., Fugelsang, J. A., \& Koehler, D. J. (2015). What makes us think? A three-stage dual-process model of analytic engagement. Cognitive Psychology, 80, 34-72. http://dx.doi.org/10.1016/j.cogpsych.2015 .05 .001 
Reber, R., \& Unkelbach, C. (2010). The epistemic status of processing fluency as source for judgments of truth. Review of Philosophy and Psychology, 1, 563-581. http://dx.doi.org/10.1007/s13164-010-0039-7

Reber, R., Winkielman, P., \& Schwarz, N. (1998). Effects of perceptual fluency on affective judgments. Psychological Science, 9, 45-48. http:// dx.doi.org/10.1111/1467-9280.00008

Rouder, J. N., Morey, R. D., Speckman, P. L., \& Province, J. M. (2012). Default Bayes factors for ANOVA designs. Journal of Mathematical Psychology, 56, 356-374. http://dx.doi.org/10.1016/j.jmp.2012.08.001

Schwarz, N., \& Clore, G. L. (1983). Mood, misattribution, and judgments of well-being: Informative and directive functions of affective states. Journal of Personality and Social Psychology, 45, 513-523. http://dx .doi.org/10.1037/0022-3514.45.3.513

Singmann, H., Klauer, K. C., \& Kellen, D. (2014). Intuitive logic revisited: New data and a Bayesian mixed model meta-analysis. PLOS ONE, 9, e94223. http://dx.doi.org/10.1371/journal.pone.0094223

Sloman, S. A. (2014). Two systems of reasoning, an update. In J. Sherman, B. Gawronski, \& Y. Trope (Eds.), Dual process theories of the social mind. New York, NY: Guilford Press.

Strack, F., Martin, L. L., \& Stepper, S. (1988). Inhibiting and facilitating conditions of the human smile: A nonobtrusive test of the facial feedback hypothesis. Journal of Personality and Social Psychology, 54, 768-777. http://dx.doi.org/10.1037/0022-3514.54.5.768

Thompson, V. A., Prowse Turner, J. A., \& Pennycook, G. (2011). Intuition, reason, and metacognition. Cognitive Psychology, 63, 107-140. http:// dx.doi.org/10.1016/j.cogpsych.2011.06.001

Topolinski, S. (2011). A process model of intuition. European Review of Social Psychology, 22, 274-315.

Topolinski, S., \& Strack, F. (2009). The analysis of intuition: Processing fluency and affect in judgements of semantic coherence. Cognition and
Emotion, 23, 1465-1503. http://dx.doi.org/10.1080/02699930 802420745

Trippas, D., Handley, S. J., \& Verde, M. F. (2013). The SDT model of belief bias: Complexity, time, and cognitive ability mediate the effects of believability. Journal of Experimental Psychology: Learning, Memory, and Cognition, 39, 1393-1402. http://dx.doi.org/10.1037/a0032398

Trippas, D., Handley, S. J., \& Verde, M. F. (2014). Fluency and belief bias in deductive reasoning: New indices for old effects. Frontiers in Psychology, 24. http://dx.doi.org/10.3389/fpsyg.2014.00631

Verde, M. F., Stone, L. K., Hatch, H. S., \& Schnall, S. (2010). Distinguishing between mnemonic and attributional sources of familiarity: The case of positive emotion bias. Memory \& Cognition, 38, 142-153. http://dx.doi.org/10.3758/MC.38.2.142

Whittlesea, B. W. A. (1993). Illusions of familiarity. Journal of Experimental Psychology: Learning, Memory, and Cognition, 19, 1235-1253. http://dx.doi.org/10.1037/0278-7393.19.6.1235

Whittlesea, B. W. A. (2002). Two routes to remembering (and another to remembering not). Journal of Experimental Psychology: General, 131, 325-348. http://dx.doi.org/10.1037/0096-3445.131.3.325

Whittlesea, B. W. A., \& Leboe, J. P. (2003). Two fluency heuristics (and how to tell them apart). Journal of Memory and Language, 49, 62-79. http://dx.doi.org/10.1016/S0749-596X(03)00009-3

Winkielman, P., \& Cacioppo, J. T. (2001). Mind at ease puts a smile on the face: Psychophysiological evidence that processing facilitation elicits positive affect. Journal of Personality and Social Psychology, 81, 9891000. http://dx.doi.org/10.1037/0022-3514.81.6.989

Received February 16, 2015

Revision received December 19, 2015

Accepted December 30, 2015 